\title{
Identification of pseudobulbar affect symptoms in Veterans with possible traumatic brain injury
}

\author{
Jennifer R. Fonda, MA; ${ }^{1-2 *}$ Phillip R. Hunt, ScD; ${ }^{3}$ Regina E. McGlinchey, PhD; ${ }^{1,4}$ James L. Rudolph, MD, \\ SM; ${ }^{1,5}$ William P. Milberg, PhD $;{ }^{1,4}$ Matthew W. Reynolds, PhD ${ }^{3}$ Charles Yonan, PharmD ${ }^{6}$ \\ ${ }^{1}$ Translational Research Center for Traumatic Brain Injury and Stress Disorders, and Geriatric Research, Education \\ and Clinical Center, Department of Veterans Affairs Boston Healthcare System, Boston, MA; ${ }^{2}$ Department of Epidemi- \\ ology, Boston University School of Public Health, Boston, MA; ${ }^{3}$ Retrospective Observational Studies, Evidera, Lexing- \\ ton, MA; Departments of ${ }^{4}$ Psychiatry and ${ }^{5}$ Medicine, Harvard Medical School, Boston, MA; ${ }^{6}$ Health Economics and \\ Outcomes Research, Avanir Pharmaceuticals Inc, Aliso Viejo, CA
}

\begin{abstract}
Pseudobulbar affect (PBA), a neurological syndrome characterized primarily by involuntary episodes of laughing and crying, can develop secondary to neurological conditions including traumatic brain injury (TBI). Veterans of the wars in Afghanistan and Iraq have an unprecedented risk for TBI, primarily from blast-related munitions. In this crosssectional study with linkage to Department of Veterans Affairs (VA) clinical data, Veterans screening positive for TBI on the VA TBI screen $(N=4,282)$ were mailed packets containing two PBA symptom assessments: a single PBA symptom screen question and the Center for Neurologic Study-Lability Scale (CNS-LS) questionnaire. Seventy percent $(n=513)$ of the 728 Veteran respondents screened positive for PBA symptoms with a CNS-LS score of 13 or greater. There was strong concordance between PBA symptom prevalence measured with the single screening question and CNS-LS, with high sensitivity $(0.87)$ and positive predictive value $(0.93)$ and moderate specificity (0.79). Posttraumatic stress disorder (54\% vs 32\%), major depression ( $35 \%$ vs $22 \%$ ), and anxiety disorder (20\% vs $13 \%$ ) were more common for Veterans with PBA symptoms than for those without. PBA symptoms were common in this Veteran cohort, were detected using simple screening tools, and often co-occurred with other psychiatric disorders common in Veterans.
\end{abstract}

Key words: cross-sectional surveys, depression, emotional lability, lability scale, nervous system diseases, pseudobulbar affect, pseudobulbar syndromes, PTSD, traumatic brain injuries, Veterans.

\section{INTRODUCTION}

Pseudobulbar affect (PBA), a neurological syndrome characterized by involuntary, uncontrollable, exaggerated, and often inappropriate outbursts of crying and/or laughing, can cause severe distress, embarrassment, and

\footnotetext{
Abbreviations: $\mathrm{ALS}=$ amyotrophic lateral sclerosis, $\mathrm{AUC}=$ area under the ROC curve, $\mathrm{CDW}=$ Corporate Data Warehouse, CNS-LS = Center for Neurologic Study-Lability Scale, ICD-9 = International Classification of Diseases-Ninth Revision, $\mathrm{MS}=$ multiple sclerosis, NewGen $=$ New Generation of U.S. Veterans, OEF = Operation Enduring Freedom, OIF = Operation Iraqi Freedom, OND = Operation New Dawn, PBA $=$ pseudobulbar affect, $\mathrm{PLC}=$ pathological laughing and crying, $\mathrm{PPV}=$ positive predictive value, $\mathrm{PTSD}=$ posttraumatic stress disorder, $\mathrm{ROC}=$ receiver operating characteristic, $\mathrm{SD}=$ standard deviation, $\mathrm{Se}=$ sensitivity, $\mathrm{Sp}=$ specificity, $\mathrm{SSN}=$ Social Security number, TBI = traumatic brain injury, TRACTS = Translational Research Center for TBI and Stress Disorders, VA = Department of Veterans Affairs, VHA = Veterans Health Administration, VISN = Veterans Integrated Service Network.

*Address all correspondence to Jennifer R. Fonda, PhD; TRACTS/GRECC 182 (JP), VA Boston Healthcare System, 150 South Huntington Ave, Boston, MA 02130; 857-3646677; fax: 857-364-4544. Email: jennifer.fonda@va.gov http://dx.doi.org/10.1682/JRRD.2014.08.0191
} 
social dysfunction [1]. This syndrome develops in the context of numerous neurological conditions, including stroke, multiple sclerosis (MS), amyotrophic lateral sclerosis (ALS), Parkinson disease, Alzheimer disease, and traumatic brain injury (TBI) [2-4]. PBA is believed to be caused by disruption or damage to the neural circuitry between the cerebellum and frontal cerebral cortex, which modulates emotional expression [1,4-7]. In the PBA Registry Series, a large $(N=5,290)$ clinical sample of patients with neurological conditions associated with PBA, the prevalence of PBA symptoms as defined by a score of 13 or greater in the Center for Neurologic StudyLability Scale (CNS-LS) [5] was approximately 37 percent [2]. The prevalence of moderate or severe PBA symptoms, defined as CNS-LS score $\geq 21$, was 9 percent and the highest among the 590 patients with TBI $(n=96$, $16 \%$ ). This rate was similar to what was reported by Tateno et al. regarding the prevalence of pathological laughing and crying (PLC), an alternate term for PBA [8]. PLC following TBI was reported in approximately 11 percent of a sample of patients admitted for a closed head injury. Furthermore, patients with PLC had more depressive, anxious, and aggressive behaviors than patients without PLC. PLC was also associated with anxiety disorder and disruption of frontal lobe function [8].

Servicemembers returning from Operation Iraqi Freedom (OIF), Operation Enduring Freedom (OEF), and Operation New Dawn (OND) in Iraq and Afghanistan have been exposed to unprecedented risk for TBI due primarily to high rates of exposure to blast-related munitions. Blasts and explosions are the most common cause of injury for U.S. military personnel engaged in OIF/ OEF/OND [9], and the reported incidence of TBI in OIF/ $\mathrm{OEF} / \mathrm{OND}$ Veterans ranges from 9 to 39 percent [10-13]. It is currently unknown whether these cases of TBI are associated with the development of PBA symptoms.

The current study had two primary aims: first, to estimate the occurrence of PBA symptoms in OIF/OEF/ OND Veterans using two survey instruments mailed to Veterans who screened positive for TBI, and second, to evaluate the concordance between the two survey instruments used to assess the occurrence of PBA symptomsa single PBA screen question [5] and the CNS-LS questionnaire [6]. To date, there is not a standard method to screen for PBA specifically in TBI.

\section{METHODS}

\section{Study Population}

Our study population consisted of OIF/OEF/OND Veterans in the Department of Veterans Affairs (VA) New England Healthcare System, Veterans Integrated Service Network (VISN)-1, who screened positive for TBI on the Veterans Health Administration (VHA) four-item TBI screen between April 2007 and April 2013. We excluded Veterans who had an International Classification of Diseases-Ninth Revision (ICD-9) diagnosis code for bipolar disorder (296.0, 296.1, and 296.4-296.8), schizophrenia, or other psychiatric disorder $(293.81,293.82,295$, and 297-298) recorded at any time in their VA medical record. Veterans with a diagnosis of psychosis not otherwise specified due to trauma-related hallucinations (293.9) were retained in the study.

\section{Procedure}

We obtained names and mailing addresses from the VA Corporate Data Warehouse (CDW) Patient Data Domain. All Veterans in our study population were mailed a packet that included a cover letter, two questionnaires, and a pre-addressed, postage-paid return envelope. The cover letter included all elements of consent, informing the Veteran that completion of the questionnaire was considered implied consent. The first questionnaire included the seven-item CNS-LS, supplemented with a single additional PBA screening question (see "Measures" section). The EuroQol 5-dimension 5-level questionnaire was also included as a health status measure (subject of future publication). Each survey contained a unique study code to maintain confidentiality. Survey responses were hand-entered into a database and further verified for quality assurance. The survey data were linked to the VHA files using a crosswalk file that contained the study code and scrambled Social Security number (SSN), which is a unique identifier for each Veteran.

Baseline demographic and clinical characteristics were obtained from the VHA electronic medical data sets during VA fiscal year 2012 (October 1, 2011-September 30, 2012). Data for inpatient hospitalizations and outpatient visits were extracted from the National Patient Care Database, which includes patient demographic information, the name of the clinic where the service was provided, and up to 13 ICD-9 coded diagnoses. Pharmacy data were extracted from the VA Decision Support System pharmacy files, which include all outpatient prescription orders filled at a VA pharmacy or consolidated mail 
outpatient pharmacy. Laboratory data were extracted from the VA CDW, which includes the laboratory test name, result, and unit. All files were merged by scrambled SSN. All procedures were approved by the VA Boston Institutional Review Board. Burden of disease and comorbidities, quality of life, and costs in patients with PBA symptoms will be reported elsewhere.

\section{Measures}

\section{Traumatic Brain Injury Assessment}

The VA TBI screen, instituted in April 2007, is a onetime mandatory screening for all Veterans entering the VA medical system who served in OIF/OEF/OND [14]. This screen, which is based on the Brief Traumatic Brain Injury Screen [15], consists of four sequential questions assessing-

1. Events associated with the risk for TBI (e.g., blast, fall).

2. Immediate symptoms following the event (e.g., confusion, being dazed).

3. New or worsening symptoms following the event (e.g., dizziness, headaches)

4. Symptoms within the past week (e.g., headaches, sleep problems).

Results from the screen are recorded in the VA CDW Patient Data Domain, from which we identified OIF/OEF/ OND Veterans who screened positive on the VHA 4-item TBI screen. Recently, Fortier et al. reported high sensitivity $(\mathrm{Se}=0.85)$ and specificity $(\mathrm{Sp}=0.82)$ of the VA screen for predicting probable cases of TBI when administered for research purposes, although sensitivity was lower when compared with a semistructured clinical interview (Boston Assessment of Traumatic Brain Injury-Lifetime) [10]. The VA screen has also been shown to have high internal consistency and reliability [16].

If a Veteran answers affirmatively to all four questions on the screen, he or she is classified as probable TBI and referred for a comprehensive, secondary screening. The secondary screen follows a standard protocol administered by a clinician, with questions about the mechanism (e.g., blast or blunt trauma) and severity of the injury, as well as current neurobehavioral symptoms and psychiatric history. At the end of examination, the clinician is required to confirm or rule out a TBI diagnosis [14].

\section{Assessment of Pseudobulbar Affect Symptoms}

We assessed the presence of PBA symptoms using the CNS-LS questionnaire supplemented with a single
PBA screening question. The 7-item CNS-LS is a quantitative measure of the frequency and severity of involuntary or excessive laughing and crying symptoms [6]. Possible responses to each question are as follows: (1) applies never, (2) applies rarely, (3) applies occasionally, (4) applies frequently, and (5) applies most of the time. The total score is the sum of the individual question responses and ranges from 7 (no symptoms) to 35 (highest frequency/severity). The CNS-LS was also divided into laughing and crying subscales. The laughing subscale consists of four questions, and the crying subscale consists of three questions [6]. The CNS-LS has been validated as a measure of PBA symptoms in patients with ALS and MS [5-6,17]; however, it has not been specifically validated in patients with TBI. The single PBA symptom screening question was included to assess the CNS-LS performance. The PBA symptom screening question asks the Veteran, "Have you ever experienced involuntary episodes of crying and/or laughing that were exaggerated or even contrary to how you felt at the time?" Possible responses for this screening question are "yes," "no," and "unsure." The single question was used in a recently published PBA symptom prevalence survey [5]. In that study, of the respondents with a CNS-LS score of 13 or greater, 70 percent answered "yes" to the symptom screening single question.

\section{Statistical Analyses}

The presence of PBA symptoms was assessed separately using the two screening instruments. Veterans were categorized as screening positive with symptoms for PBA if they had a CNS-LS score of 13 or greater or if they answered "yes" on the single PBA screening question. We assessed the concordance between the two survey instruments to determine the proportion that screened positive for PBA symptoms on both measures. Additionally, Veterans were categorized as screening positive for moderate or severe PBA symptoms if they had a CNS-LS score of 21 or greater. We characterized the study population overall and by PBA symptom screening status (positive and negative for symptoms by each of the two screening instruments) by demographic, clinical, and TBI characteristics obtained from the previous fiscal year (October 1, 2011-September 30, 2012). The descriptive statistics included means and standard deviations (SDs) for continuous variables and frequencies and percentage for categorical variables. 
The distribution of CNS-LS responses was tabulated and stratified by CNS-LS subscale and PBA symptom single screening question response. The mean and SD for the CNS-LS total score and frequency and percent for the PBA symptom screening question were computed. Statistical significance of the difference in CNS-LS total score between Veterans with positive and negative PBA symptom screening responses was determined using a $t$-test. Linear trends of CNS-LS question responses with PBA symptom single screening question responses were tested using Pearson correlation coefficient and log-linear association models [18] for each of the CNS-LS questions separately. A significant, positive trend test indicates that the proportion who responded "yes" on the PBA symptom single screening question increases linearly with increasing CNS-LS category responses.

Analogs of sensitivity and specificity of the CNS-LS total scores at various thresholds relative to the PBA symptom screening question were determined and used to develop the receiver operating characteristic (ROC) curves. True measures of sensitivity and specificity are not calculable because neither the CNS-LS nor the single PBA symptom screening question are validated measures of PBA symptoms in a population with TBI. The area under the ROC curve (AUC) is an index of the accuracy of the dichotomous test on a probability scale of 0 to 1 . An AUC of 1 indicates a perfect test, and an AUC of 0.5 indicates a test with no ability to discriminate positive from negative results [19]. Separate ROCs were constructed using the CNS-LS total score and the laughing and crying subscales. The analogs of sensitivity, specificity, and positive predictive value (PPV) are reported for each ROC analysis.

Analyses were conducted using SAS (version 9.3; Cary, North Carolina) software. All statistical tests were two-sided, using $p<0.05$ as the cutoff for reporting statistically significant results.

\section{RESULTS}

\section{Demographic and Clinical Characteristics}

Veterans with Positive Traumatic Brain Injury Screen

We identified 33,487 OIF/OEF/OND Veterans in the VISN-1 who received the VA primary TBI screen between April 2007 and April 2013, of whom 4,837 (14\%) screened positive for TBI. We excluded 437 Veter- ans with a diagnosis for bipolar disorder or schizophrenia and 118 with incomplete or duplicate demographic information. Our final sample was 4,282 Veterans who screened positive for TBI.

Our study population was predominantly male $(95 \%)$, with an average age of $34.5 \pm 8.8 \mathrm{yr}$. This sample closely resembles the demographic characteristics of the National Health Study for a New Generation of U.S. Veterans (NewGen), which is a national cohort of OIF/OEF Veterans and nondeployed Veterans who served during the same era [20]. The key difference was that our sample had a higher proportion of males than the NewGen cohort (95\% vs $88 \%$ ). The complete demographic characteristics are presented in Table 1.

In the screened TBI study population, about half had at least one current type of pain condition, with back and neck pain (32\%), other arthropathies (31\%), and headaches and migraines (17\%) as the most common (Table 2). Current mental health conditions were highly prevalent in this population, including posttraumatic stress disorder (PTSD) (46\%), major depression (26\%), and anxiety disorders (17\%). Within the year 2012, more than a third of the population had at least one prescription for an antidepressant (37\%), 16 percent for a sedative or hypnotic agent, and 7 percent for an antipsychotic (in spite of psychosis being an exclusion criterion) (Table 2).

Blast or explosion was the most common mechanism of injury (84\%) reported on the TBI screen among Veterans who screened positive for TBI, followed by fall (46\%), other mechanism (46\%), and vehicle accident (42\%). It is important to note that the mechanisms of injury are not mutually exclusive; a Veteran could report more than one mechanism on the VHA TBI screen if he or she experienced more than one probable TBI, or more than one mechanism of injury could be reported for a single TBI event (e.g., blast and fall). The number of possible TBI events is not assessed in the screen. Finally, in the subset of $2,467(58 \%)$ who completed the secondary TBI evaluation, 1,311 (53\%) had a confirmed TBI. The majority of the confirmed TBIs were mild (89\%).

\section{Comparison of Respondents and Nonrespondents}

There were 758 Veterans (19\%) who completed the survey among the subset of 3,954 (92\%) who had accurate mailing addresses. Compared with nonrespondents, respondents were more likely to be older, white, married, and college graduates (Table 1). Respondents had a higher prevalence of depression and were more likely to 
Table 1.

Baseline demographic characteristics, stratified by responder and pseudobulbar affect (PBA) symptom status.

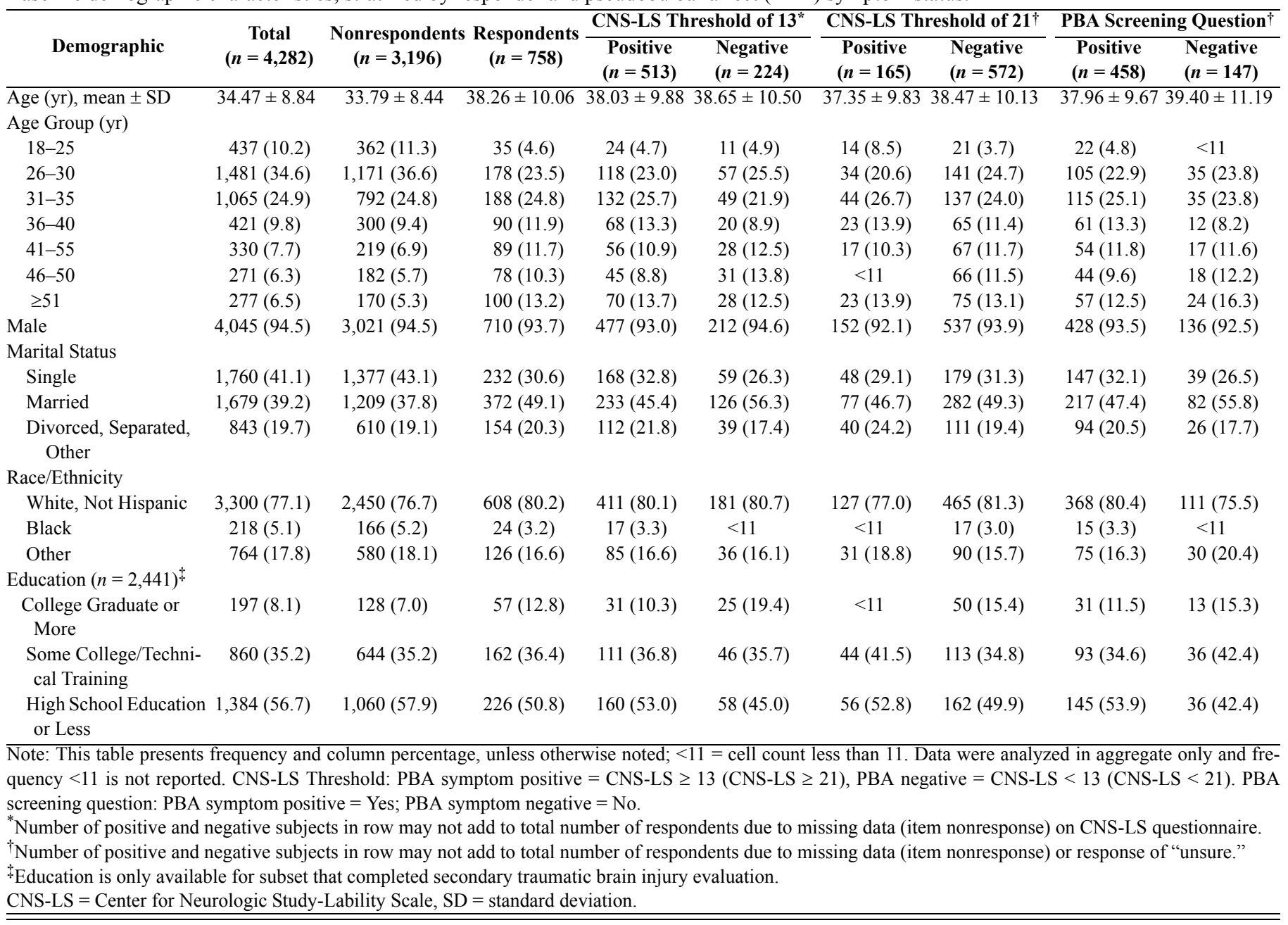

have a prescription for antidepressants than nonrespondents. Additionally, respondents were more likely to have a prescription for antiepileptics than nonrespondents (Table 2). Finally, respondents also had a higher prevalence of pain-related diagnoses, mostly due to higher prevalence of osteoarthritis and other arthropathies. Respondents were similar to nonrespondents for all other comorbid conditions and biomedical characteristics, including PTSD, anxiety disorders, substance abuse, and headaches/migraines (Tables 2 and Appendix Table S1, available online only).

\section{Center for Neurologic Study-Lability Scale Overall Score}

Nearly all respondents $(97 \%)$ completed every item on the CNS-LS questionnaire. Of these, the average CNS-LS score was $16.0 \pm 5.7$, with the majority of Veterans $(70 \%)$ screening positive for PBA symptoms with a CNS-LS score of 13 or greater and 22 percent screening positive for moderate or severe PBA symptoms (CNS-LS $\geq 21 ; n=165$ ). For the PBA symptom screening single question, 60 percent answered "yes," 20 percent answered "no," 14 percent answered "unsure," and 6 percent did not answer.

In the subset of 591 respondents $(78 \%)$ with complete data for the CNS-LS questions and a "yes" or "no" answer for the PBA symptom screening question, 62 percent screened positive and 20 percent screened negative for PBA symptoms on both measures; 21.3 percent had a CNS-LS score of 21 or greater and a "yes" response on the screening question. Greater than 99 percent $(126 / 127)$ of those with CNS-LS score of 21 or greater answered "yes" to the screening questions. Those who responded "yes" on the PBA symptom screening question had a significantly higher average CNS-LS score than those who answered "no" $(18.4 \pm 5.0$ vs $10.1 \pm 3.4$, respectively; $p<$ 
Table 2.

Mental health and traumatic brain injury characteristics, stratified by responder and pseudobulbar affect (PBA) symptom status.

\begin{tabular}{|c|c|c|c|c|c|c|c|c|c|}
\hline \multirow[b]{2}{*}{ Characteristic } & \multirow{2}{*}{$\begin{array}{c}\text { Total } \\
(n=\mathbf{4 , 2 8 2})\end{array}$} & \multirow{2}{*}{$\begin{array}{l}\text { Nonrespondents } \\
\quad(n=3,196)\end{array}$} & \multirow{2}{*}{$\begin{array}{l}\text { Respondents } \\
\quad(n=758)\end{array}$} & \multicolumn{2}{|c|}{ CNS-LS Threshold of $13^{*}$} & \multicolumn{2}{|c|}{ CNS-LS Threshold of $21^{*}$} & \multicolumn{2}{|c|}{ PBA Screening Question } \\
\hline & & & & $\begin{array}{c}\text { Positive } \\
(n=513) \\
\end{array}$ & $\begin{array}{l}\text { Negative } \\
(n=224)\end{array}$ & $\begin{array}{c}\text { Positive } \\
(n=165) \\
\end{array}$ & $\begin{array}{l}\text { Negative } \\
(n=572)\end{array}$ & $\begin{array}{c}\text { Positive } \\
(n=458)\end{array}$ & $\begin{array}{l}\text { Negative } \\
(n=147)\end{array}$ \\
\hline \multicolumn{10}{|c|}{ TBI Severity $(n=1,311) \stackrel{\dagger}{+}$} \\
\hline Moderate or Severe & $147(11.2)$ & $114(11.6)$ & $23(9.6)$ & $18(10.5)$ & $<11$ & $<11$ & $15(9.1)$ & $15(10.1)$ & $<11$ \\
\hline \multicolumn{10}{|l|}{ Mechanism of Injury } \\
\hline Blast or Explosion & $3,608(84.3)$ & $2,684(84.0)$ & $629(83.0)$ & $432(84.2)$ & $179(79.9)$ & $137(83.0)$ & $474(82.9)$ & $378(82.5)$ & $120(81.6)$ \\
\hline Fall & $1,952(45.6)$ & $1,457(45.6)$ & $362(47.8)$ & $252(49.1)$ & $100(44.6)$ & $84(50.9)$ & $268(46.9)$ & $231(50.4)$ & $58(39.5)$ \\
\hline Vehicle Accident & $1,804(42.1)$ & $1,356(42.4)$ & $317(41.8)$ & $212(41.3)$ & $94(42.0)$ & $71(43.0)$ & $235(41.1)$ & $193(42.1)$ & $57(38.8)$ \\
\hline Other & $1,961(45.8)$ & $1,469(46.0)$ & $342(45.1)$ & $238(46.4)$ & $91(40.6)$ & $79(47.9)$ & $250(43.7)$ & $218(47.6)$ & $68(46.3)$ \\
\hline Pain Condition & $2,157(50.4)$ & $1,623(50.8)$ & $410(54.1)$ & $284(55.4)$ & $112(50.0)$ & $97(58.8)$ & $299(52.3)$ & $252(55.0)$ & $76(51.7)$ \\
\hline Osteoarthritis & $243(5.7)$ & $165(5.2)$ & $66(8.7)$ & $48(9.4)$ & $17(7.6)$ & $13(7.9)$ & $52(9.1)$ & $42(9.2)$ & $11(7.5)$ \\
\hline Back/Neck Pain & $1,352(31.6)$ & $1,033(32.3)$ & $254(33.5)$ & $173(33.7)$ & $71(31.7)$ & $62(37.6)$ & $182(31.8)$ & $154(33.6)$ & $41(27.9)$ \\
\hline Major Depression & $1,117(26.1)$ & $817(25.6)$ & $235(31.0)$ & $177(34.5)$ & 49 (21.9) & $60(36.4)$ & $166(29.0)$ & $149(32.5)$ & $32(21.8)$ \\
\hline PTSD & $1,963(45.8)$ & $1,483(46.4)$ & $363(47.9)$ & $275(53.6)$ & $72(32.1)$ & $96(58.2)$ & $251(43.9)$ & $238(52.0)$ & $43(29.3)$ \\
\hline Anxiety Disorders & $721(16.8)$ & $545(17.1)$ & $136(17.9)$ & $103(20.1)$ & $29(13.0)$ & $33(20.0)$ & $99(17.3)$ & $93(20.3)$ & $21(14.3)$ \\
\hline \multicolumn{10}{|l|}{ Substance Abuse } \\
\hline Alcohol-Related & $603(14.1)$ & $450(14.1)$ & $113(14.9)$ & $85(16.6)$ & $25(11.2)$ & $28(17.0)$ & $82(14.3)$ & $79(17.3)$ & $13(8.8)$ \\
\hline Drug-Related & $403(9.4)$ & $295(9.2)$ & $73(9.6)$ & $52(10.1)$ & $17(7.6)$ & $23(13.9)$ & $46(8.0)$ & $48(10.5)$ & $<11$ \\
\hline Tobacco Use & $688(16.1)$ & $518(16.2)$ & $121(16.0)$ & $88(17.2)$ & $28(12.5)$ & $35(21.2)$ & $81(14.2)$ & $81(17.7)$ & $14(9.5)$ \\
\hline \multicolumn{10}{|l|}{ Prescription Medication } \\
\hline Antidepressants & $1,558(36.4)$ & $1,141(35.7)$ & $316(41.7)$ & $236(46.0)$ & $70(31.3)$ & $90(54.6)$ & $216(37.8)$ & $211(46.1)$ & $45(30.6)$ \\
\hline Antipsychotics & $302(7.1)$ & $222(6.9)$ & $58(7.7)$ & $44(8.6)$ & $<11$ & $19(11.5)$ & $35(6.1)$ & $39(8.5)$ & $<11$ \\
\hline Opioids & $609(14.2)$ & $450(14.1)$ & $117(15.4)$ & 97 (18.9) & $17(7.6)$ & $34(20.6)$ & $80(14.0)$ & $75(16.4)$ & $13(8.8)$ \\
\hline
\end{tabular}

Note: This table presents frequency and column percentage, unless otherwise noted; $<11=$ cell count less than 11 . Data were analyzed in aggregate only and frequency $<11$ is not reported.

"Number of positive and negative subjects in row may not add to total number of respondents due to missing data (item nonresponse) on CNS-LS questionnaire.

†Number of positive and negative subjects in row may not add to total number of respondents due to missing data (item nonresponse) or response of "unsure."

\#TBI severity is based on evaluation according to accepted Department of Veterans Affairs and Department of Defense criteria based on duration of alternation of consciousness, posttraumatic amnesia, or loss of consciousness for most severe TBI during deployment. Only subset who completed secondary TBI evaluation have at least one confirmed TBI and completed severity questions are included in table for TBI severity.

CNS-LS = Center for Neurologic Study-Lability Scale, PTSD = posttraumatic stress disorder, TBI = traumatic brain injury.

0.001). All CNS-LS questions showed a significant increasing linear trend of symptom frequency with a "yes" response on the PBA symptom screening question $(p<0.001 ;$ Appendix Table S2).

\section{Evaluation of Center for Neurologic Study-Lability Scale Questionnaire and Pseudobulbar Affect Symptom Screening Question}

The ROC analysis indicated that the optimum analogs of sensitivity and specificity occurred at the CNS-LS total score of $12(\mathrm{Se}=0.87, \mathrm{Sp}=0.79, \mathrm{PPV}=0.93$; Table 3). The AUC was 0.914 , indicating that the single
PBA symptom screening question discriminates very well between those with and without PBA symptoms as determined by the CNS-LS.

CNS-LS responses showed that reported crying symptoms outweighed laughing symptoms (Table 4). The study population weighted mean score (laughing scores weighted by 0.75 ) for the CNS-LS crying questions (questions 1, 3, and 6) was significantly higher than the weighted mean score for the laughing questions (questions $2,4,5$, and 7) (7.5 \pm 3.1 vs $6.4 \pm 2.7$, respectively; $p<0.001$ ). 
Table 3.

Concordance between Center for Neurologic Study-Lability Scale (CNS-LS) questionnaire threshold and pseudobulbar affect (PBA) symptom screening question $(N=591), n(\%)$.

\begin{tabular}{lccc}
$\begin{array}{l}\text { PBA Symptom } \\
\begin{array}{c}\text { Screening } \\
\text { Question }\end{array}\end{array}$ & $\begin{array}{c}\text { CNS-LS } \\
\text { Positive }\end{array}$ & $\begin{array}{c}\text { CNS-LS } \\
\text { Negative }\end{array}$ & Total \\
Yes & $390(66.0)$ & $56(9.5)$ & $446(75.5)$ \\
No & $30(5.1)$ & $115(19.5)$ & $145(24.5)$ \\
Total & $420(71.1)$ & $171(28.9)$ & $591(100)$ \\
\hline $\begin{array}{l}\text { Note: CNS-LS threshold } \geq 12 . \text { Sensitivity }=0.87, \text { Specificity }=0.79, \text { positive } \\
\text { predictive value }=0.93 .\end{array}$ \\
\hline
\end{tabular}

The ROC analyses indicate that the PBA symptom single screening question discriminates CNS-LS cryingrelated symptoms better than it does CNS-LS laughingrelated symptoms (AUC $=0.908$ and 0.806 , respectively).

\section{DISCUSSION}

Self-report of PBA symptoms was common among OIF/OEF/OND Veterans who screened positive for TBI and responded to our survey. There was high concordance between the PBA symptom single screening question and PBA symptoms measured on the CNS-LS 7-item rating scale. The ROC analysis indicated that a CNS-LS threshold of 12 provided optimal discrimination between positive and negative responses on the PBA symptom screening question, with high sensitivity and PPV and moderate specificity. The CNS-LS has been validated as a measure of PBA symptoms in patients with ALS and MS [5-6,17]. In ALS patients, a CNS-LS score of 13 or greater provided the highest incremental validity for physician diagnosis of PBA with a sensitivity of 0.84 and specificity of 0.81 . Among MS patients, the optimal threshold corresponding to physician diagnosis of PBA for the CNS-LS was reported as 17 or greater [17]. For both ALS and MS patients, a threshold of CNS-LS $\geq 13$ was found to provide good predictive value (PPV $=0.82$ and 0.78 , respectively) and good sensitivity $(\mathrm{Se}=0.84$ and 0.96 , respectively) $[6,17]$ but poor specificity in the MS patients $(\mathrm{Sp}=0.55)$. In this study, the analogs of sensitivity and specificity were not significantly different when using a threshold of CNS-LS $\geq 13(\mathrm{Se}=0.82, \mathrm{Sp}=0.83)$, rather than the optimal threshold of CNS-LS $\geq 12$, and were very similar to those reported for ALS patients [6]. This consistency across populations suggests that the CNS-LS is a reliable screen for PBA symptoms, as shown in other studies $[6,17]$ and further, that the PBA symptom single screening question can be used in Veterans as a sensitive and specific screening tool for CNS-LS-defined PBA symptoms.

The CNS-LS has not been validated in a population with TBI and may not be sufficiently specific to discriminate PBA from other concomitant disorders that can be associated with affective lability or crying, such as PTSD or depression, which are highly prevalent in this study

Table 4.

Responses for each item on Center for Neurologic Study-Lability Scale questionnaire $(N=758), n(\%)$.

\begin{tabular}{|c|c|c|c|c|c|c|}
\hline Item & $\begin{array}{c}\text { Applies } \\
\text { Never (1) }\end{array}$ & $\begin{array}{c}\text { Applies } \\
\text { Rarely (2) }\end{array}$ & $\begin{array}{c}\text { Applies } \\
\text { Occasionally (3) }\end{array}$ & $\begin{array}{c}\text { Applies } \\
\text { Frequently (4) }\end{array}$ & $\begin{array}{l}\text { Applies Most of } \\
\text { the Time (5) }\end{array}$ & $\begin{array}{c}\text { No } \\
\text { Response }\end{array}$ \\
\hline $\begin{array}{l}\text { 1. There are times when I feel fine one minute, } \\
\text { and then I'll become tearful the next over } \\
\text { something small or for no reason at all. }\end{array}$ & $149(19.7)$ & $147(19.4)$ & $260(34.3)$ & $164(21.6)$ & $35(4.6)$ & $3(0.4)$ \\
\hline $\begin{array}{l}\text { 2. Others have told me that I seem to become } \\
\text { amused very easily or that I seem to become } \\
\text { amused about things that really aren't funny. }\end{array}$ & $232(30.6)$ & $164(21.6)$ & $210(27.7)$ & $118(15.6)$ & $30(4.0)$ & $4(0.5)$ \\
\hline 3. I find myself crying very easily. & $184(24.3)$ & $197(26.0)$ & $193(25.5)$ & $138(18.2)$ & $39(5.2)$ & $7(0.9)$ \\
\hline $\begin{array}{l}\text { 4. I find that even when I try to control my } \\
\text { laughter, I am often unable to do so. }\end{array}$ & $271(37.8)$ & $273(36.0)$ & $130(17.2)$ & $59(7.8)$ & $15(2.0)$ & $10(1.3)$ \\
\hline $\begin{array}{l}\text { 5. There are times when I won't be thinking of } \\
\text { anything happy or funny at all, but then I'll } \\
\text { suddenly be overcome by funny or happy } \\
\text { thoughts. }\end{array}$ & $286(37.7)$ & $219(28.9)$ & $167(22.0)$ & $65(8.6)$ & $18(2.4)$ & $3(0.4)$ \\
\hline $\begin{array}{l}\text { 6. I find that even when I try to control my } \\
\text { crying, I am often unable to do so. }\end{array}$ & $251(33.1)$ & $212(28.0)$ & $170(22.4)$ & $90(11.9)$ & $31(4.1)$ & $4(0.5)$ \\
\hline 7. I find that I am easily overcome by laughter. & $295(38.9)$ & $232(30.6)$ & $151(19.9)$ & $61(8.1)$ & $16(2.1)$ & $3(0.4)$ \\
\hline
\end{tabular}


population. Given the high occurrence of PBA symptoms in this study, it may be that the CNS-LS is not sufficiently specific to be diagnostic for PBA symptoms in this population. The single screening question, inquiring about the hallmark symptoms of PBA, was included in the survey, in part to assess specificity of the CNS-LS for PBA symptoms. So it is encouraging that the large majority of those with CNS-LS $\geq 13$ also answered "yes" to this question. Future studies should evaluate the presence of clinically diagnosed PBA in those with positive screen.

In order to maximize the number of respondents for the PBA symptom questionnaire, this study included all Veterans who screened positive on the VA primary TBI screen. False positive screens for TBI may occur if the Veteran has other conditions, including PTSD or other conditions that have concussion-like symptoms, such as hearing loss and vestibular changes [21]. The proportion of screened TBI Veterans who completed a secondary TBI assessment and subsequently received a physician diagnosis of TBI was 53 percent, which is consistent with the proportion of approximately 60 percent found in other studies [22-23]. The reported symptom prevalence was similar in both the screened TBI and the confirmed TBI groups (70\% vs $72 \%)$. Parallel analyses in the subset of Veterans with definitive TBI confirmed by a follow-up neuropsychiatric examination yielded very similar results to those reported in this larger sample of Veterans who screened positive for TBI based on the four-question screen (data not shown).

To our knowledge, this is the first study to evaluate the occurrence of PBA symptoms in a Veteran population with possible and confirmed TBI. The high prevalence of PBA symptoms in this cohort was surprising, especially given that the majority of TBIs in the current Veteran population are mild in severity. PBA symptoms are not routinely assessed in patients with mild TBI, but these data suggest that they should be, because this problem may be even more common than other comorbidities, including PTSD, depression, and anxiety disorders. Not all Veterans who screened positive for TBI were confirmed through secondary screening to have TBI (47\%), and it may be that PBA symptoms are also highly prevalent in Veterans with whom TBI is suspected but not diagnosed. As noted previously, our study found similar results in both the confirmed and unconfirmed TBI cases, suggesting that the population of Veterans of concern for PBA symptoms may be broader than just those with confirmed TBI.
Symptoms of PBA can be extremely disabling in any clinical population but perhaps even more so in Veterans who are, by their nature, very proud and confident individuals. Our data indicate that Veterans who expressed symptoms of PBA also experienced a lower quality of life and higher costs than Veterans without such symptoms (subject of future publication). We further speculate that not being in control of the basic emotional expressions of laughing and, especially, crying may be particularly distressing in this population and may add to the risk of well-documented self-destructive behaviors, including substance abuse, dangerous driving, and suicide, all of which have increased incidence in this cohort of OIF/OEF/OND Veterans [24-25]. The data here suggest that clinicians should regularly screen for PBA if there is even a possibility that the Veteran may have sustained a TBI while in service, because over half of Veterans were found to have symptoms of PBA. Our data indicate that a simple question asking a patient about involuntary episodes of laughing or crying was equally effective in assessing the presence/absence of PBA symptoms as the CNS-LS. Thus, a simple question can potentially detect the presence of a debilitating disorder that Veterans may be reluctant to talk about with a healthcare provider. When a patient answers "yes" to this question, the clinician can conduct a more in-depth probe to determine the presence and severity of potential PBA diagnosis.

It is important to recognize that TBI rarely exists in isolation in the OIF/OEF/OND Veteran. The majority of TBIs are the result of exposure to explosive munitions, which are simultaneously physically and psychologically traumatic, leading to polymorbid psychological and physical injuries. Following deployment, this is reflected in the fact that those with a history of TBI are commonly diagnosed with other conditions, including PTSD, depression, pain, and sleep disturbance [26]. To this list we now add symptoms of PBA. Because these disorders share highly interactive underlying neurological circuitry, including frontal lobe executive systems (controlling decision making, impulsivity, addiction behaviors, etc.) [27] and cerebellar modulating systems (associative learning, emotional regulation) [28], it is likely that treatment must be multifaceted and not targeted to a single condition. We suspect that this may be the reason that a substantial proportion of Veterans report PBA symptoms despite use of antidepressant medications. It is essential to treat the "whole" Veteran if clinicians are going to be effective. 
Gaining a better understanding of how one condition interacts with or compounds the burden of another will be critical to designing effective - i.e., individualized - treatments for returning servicemembers and Veterans.

This study employed a cross-sectional study design, and thus we are unable to determine the causal directional of the association between TBI and PBA symptoms. However, this study is able to minimize potential retrospective reporting biases common to cross-sectional studies by obtaining clinical and TBI information from electronic medical records and standardized TBI reports.

Another limitation of this study was the inclusion of $\mathrm{OIF} / \mathrm{OEF} / \mathrm{OND}$ Veterans who received care at a VA facility in the New England region only. Nevertheless, this sample closely resembles the demographic characteristics of a national cohort of younger U.S. Veterans [20]. Thus, results could be generalized to a national sample of $\mathrm{OIF} / \mathrm{OEF} / \mathrm{OND}$ Veterans who screened positive for TBI.

Finally, the population prevalence cannot be estimated with this study design because the responding population may not be representative of the broader TBI population, which constitutes the desired denominator. Our results depend on the subjects accurately completing and returning the surveys. The responding population may be drawn more heavily from those who are more motivated to respond due to presence of PBA symptoms or other mental health comorbidities. While there were some demographic difference between the responding and nonresponding populations and the respondents were more likely to have a diagnosis and prescriptions for depression, the prevalence of other mental health comorbidities determined from clinical records (PTSD, anxiety disorder) were similar between respondents and nonrespondents. Furthermore, respondents and nonrespondents were very similar on clinical characteristics unrelated to PBA (e.g., hypertension and hyperlipidemia), suggesting that the potential biases are minimal.

\section{CONCLUSIONS}

There is a high occurrence of PBA symptoms in OIF/ OEF/OND Veterans who screen positive for TBI. It is another piece of a very complicated puzzle of trying to understand the mental and physical needs of our returning servicemembers and Veterans. Our findings are important, highlighting the presence of PBA symptoms in addition to other comorbidities. The inability to con- trol one's emotional expressions, such as laughing and crying out of social or emotional context, may be particularly debilitating in this population who are normally proud and confident individuals. This study suggests that clinicians should regularly screen for PBA symptoms in Veterans who may have sustained a TBI while in service. To that end, having a simple question asking about involuntary episodes of laughing and crying, which was equally as effective as assessing the presence or absence of PBA symptoms using the 7-item CNS-LS questionnaire, may help with feasibility of screening in everyday clinical practice. Additionally, the single question may provide signals for required differential diagnosis. Thus, clinicians can potentially detect this debilitating disorder using this simple screening question and conduct a more thorough neuropsychiatric evaluation, including the use of a measure like the CNS-LS to gauge the frequency and severity of PBA symptoms.

\section{ACKNOWLEDGMENTS}

\section{Author Contributions:}

Significant contribution to study design: C. Yonan, R. E. McGlinchey, M. W. Reynolds, P. R. Hunt, J. R. Fonda, W. P. Milberg, J. L. Rudolph. Acquisition and verification of data: J. R. Fonda.

Contributed to analysis and interpretation: J. R. Fonda, P. R. Hunt, R. E. McGlinchey.

Primary preparation of manuscript: J. R. Fonda. Critical review and editing of manuscript: P. R. Hunt, R. E. McGlinchey, C. Yonan.

Contributed critical editing of manuscript: M. W. Reynolds, W. P. Milberg, J. L. Rudolph.

Financial Disclosures: C. Yonan is an employee of Avanir Pharmaceuticals Inc, which currently markets a drug, Nuedexta, approved for the treatment of PBA. J. R. Fonda, P. R. Hunt, and M. W. Reynolds received financial support for carrying out this work from Avanir Pharmaceuticals Inc. The remaining authors did not have any conflicting interests to declare.

Funding/Support: This material was based on work supported by Avanir Pharmaceuticals Inc. Evidera provided support for the protocol and manuscript preparation, in collaboration with VA investigators. VA Boston Healthcare System and the Translational Research Center for TBI and Stress Disorders (TRACTS), which is a VA Rehabilitation Research and Development Traumatic Brain Injury Center of Excellence (grant B6796-C), are acknowledged for providing overall and infrastructural support.

Additional Contributors: We would like to thank Arkadiy Maksimovskiy, Hannah Franz, Mary Fitzgerald, and the TRACTS team for their tremendously valuable assistance in preparing and mailing the questionnaire packets. J. R. Fonda has completed a $\mathrm{PhD}$ since this study was conducted. J. L. Rudolph is now with the Center of Innovation in 
Geriatric Services (grant CIN 13-419), Providence VA Medical Center, Providence, Rhode Island.

Participant Follow-Up: The authors do not plan to inform participants of the publication of this study.

Institutional Review: All procedures were approved by the VA Boston Healthcare System Institutional Review Board and Research and Development Committee.

\section{REFERENCES}

1. Schiffer R, Pope LE. Review of pseudobulbar affect including a novel and potential therapy. J Neuropsychiatry Clin Neurosci. 2005;17(4):447-54. [PMID:16387982]. http://dx.doi.org/10.1176/jnp.17.4.447

2. Brooks BR, Crumpacker D, Fellus J, Kantor D, Kaye RE. PRISM: A novel research tool to assess the prevalence of pseudobulbar affect symptoms across neurological conditions. PLoS ONE. 2013;8(8):e72232. [PMID:23991068] http://dx.doi.org/10.1371/journal.pone.0072232

3. Work SS, Colamonico JA, Bradley WG, Kaye RE. Pseudobulbar affect: An under-recognized and under-treated neurological disorder. Adv Ther. 2011;28(7):586-601.

[PMID:21660634]

http://dx.doi.org/10.1007/s12325-011-0031-3

4. Wortzel HS, Oster TJ, Anderson CA, Arciniegas DB. Pathological laughing and crying: Epidemiology, pathophysiology and treatment. CNS Drugs. 2008;22(7):531-45. [PMID:18547124] http://dx.doi.org/10.2165/00023210-200822070-00001

5. Colamonico J, Formella A, Bradley W. Pseudobulbar affect: Burden of illness in the USA. Adv Ther. 2012;29(9): 775-98. [PMID:22941524]

http://dx.doi.org/10.1007/s12325-012-0043-7

6. Moore SR, Gresham LS, Bromberg MB, Kasarkis EJ, Smith RA. A self report measure of affective lability. J Neurol Neurosurg Psychiatry. 1997;63(1):89-93. [PMID:9221973] http://dx.doi.org/10.1136/jnnp.63.1.89

7. Parvizi J, Coburn KL, Shillcutt SD, Coffey CE, Lauterbach EC, Mendez MF. Neuroanatomy of pathological laughing and crying: A report of the American Neuropsychiatric Association Committee on Research. J Neuropsychiatry Clin Neurosci. 2009;21(1):75-87. [PMID:19359455] http://dx.doi.org/10.1176/jnp.2009.21.1.75

8. Tateno A, Jorge RE, Robinson RG. Pathological laughing and crying following traumatic brain injury. J Neuropsychiatry Clin Neurosci. 2004;16(4):426-34. [PMID:15616168] http://dx.doi.org/10.1176/jnp.16.4.426

9. Warden D. Military TBI during the Iraq and Afghanistan wars. J Head Trauma Rehabil. 2006;21(5):398-402. [PMID:16983225] http://dx.doi.org/10.1097/00001199-200609000-00004
10. Fortier CB, Amick MM, Grande L, McGlynn S, Kenna A, Morra L, Clark A, Milberg WP, McGlinchey RE. The Boston Assessment of Traumatic Brain Injury-Lifetime (BATL) semistructured interview: Evidence of research utility and validity. J Head Trauma Rehabil. 2014;29(1):89-98. [PMID:23535389] http://dx.doi.org/10.1097/HTR.0b013e3182865859

11. Hoge CW, McGurk D, Thomas JL, Cox AL, Engel CC, Castro CA. Mild traumatic brain injury in U.S. Soldiers returning from Iraq. N Engl J Med. 2008;358(5):453-63. [PMID:18234750] http://dx.doi.org/10.1056/NEJMoa072972

12. Schneiderman AI, Braver ER, Kang HK. Understanding sequelae of injury mechanisms and mild traumatic brain injury incurred during the conflicts in Iraq and Afghanistan: Persistent postconcussive symptoms and posttraumatic stress disorder. Am J Epidemiol. 2008;167(12): 1446-52. [PMID: 18424429] http://dx.doi.org/10.1093/aje/kwn068

13. Terrio H, Brenner LA, Ivins BJ, Cho JM, Helmick K, Schwab K, Scally K, Bretthauer R, Warden D. Traumatic brain injury screening: Preliminary findings in a US Army Brigade Combat Team. J Head Trauma Rehabil. 2009; 24(1):14-23. [PMID:19158592] http://dx.doi.org/10.1097/HTR.0b013e31819581d8

14. Department of Veterans Affairs. Veterans Health Administration screening and evaluation of possible traumatic brain injury in Operation Enduring Freedom (OEF) and Operation Iraqi Freedom (OIF) Veterans. VHA Directive 2010 012, 2010 Mar 8.

15. Schwab KA, Ivins B, Cramer G, Johnson W, Sluss-Tiller M, Kiley K, Lux W, Warden D. Screening for traumatic brain injury in troops returning from deployment in Afghanistan and Iraq: Initial investigation of the usefulness of a short screening tool for traumatic brain injury. J Head Trauma Rehabil. 2007;22(6):377-89. [PMID:18025970] http://dx.doi.org/10.1097/01.HTR.0000300233.98242.87

16. Donnelly KT, Donnelly JP, Dunnam M, Warner GC, Kittleson CJ, Constance JE, Bradshaw CB, Alt M. Reliability, sensitivity, and specificity of the VA traumatic brain injury screening tool. J Head Trauma Rehabil. 2011;26(6):439-53. [PMID:21386716] http://dx.doi.org/10.1097/HTR.0b013e3182005de3

17. Smith RA, Berg JE, Pope LE, Callahan JD, Wynn D, Thisted RA. Validation of the CNS emotional lability scale for pseudobulbar affect (pathological laughing and crying) in multiple sclerosis patients. Mult Scler. 2004;10(6):679-85. [PMID:15584494] http://dx.doi.org/10.1191/1352458504ms1106oa

18. Agresti A. Analysis of categorical data. Hoboken (NJ): John Wiley \& Sons; 2003. 
19. Zou KH, O’Malley AJ, Mauri L. Receiver-operating characteristic analysis for evaluating diagnostic tests and predictive models. Circulation. 2007;115(5):654-57.

[PMID:17283280]

http://dx.doi.org/10.1161/CIRCULATIONAHA.105.594929

20. Eber S, Barth S, Kang H, Mahan C, Dursa E, Schneiderman A. The National Health Study for a New Generation of United States Veterans: Methods for a large-scale study on the health of recent veterans. Mil Med. 2013;178(9):966-69. [PMID:24005544] http://dx.doi.org/10.7205/MILMED-D-13-00175

21. Iverson GL, Langlois JA, McCrea MA, Kelly JP. Challenges associated with post-deployment screening for mild traumatic brain injury in military personnel. Clin Neuropsychol. 2009;23(8):1299-1314. [PMID:19882473] http://dx.doi.org/10.1080/13854040903153902

22. Hendricks AM, Amara J, Baker E, Charns MP, Gardner JA, Iverson KM, Kimerling R, Krengel M, Meterko M, Pogoda TK, Stolzmann KL, Lew HL. Screening for mild traumatic brain injury in OEF-OIF deployed US military: An empirical assessment of VHA's experience. Brain Inj. 2013;27(2): 125-34. [PMID:23384211] http://dx.doi.org/10.3109/02699052.2012.729284

23. Ruff RL, Riechers RG 2nd, Wang XF, Piero T, Ruff SS. A case-control study examining whether neurological deficits and PTSD in combat veterans are related to episodes of mild TBI. BMJ Open. 2012;2(2):e000312.

[PMID:22431700]

http://dx.doi.org/10.1136/bmjopen-2011-000312

24. Fear NT, Iversen AC, Chatterjee A, Jones M, Greenberg N, Hull L, Rona RJ, Hotopf M, Wessely S. Risky driving among regular armed forces personnel from the United Kingdom. Am J Prev Med. 2008;35(3):230-36. [PMID:18617356] http://dx.doi.org/10.1016/j.amepre.2008.05.027

25. Tanielian T, Jaycox LH. Invisible wounds of war: Psychological and cognitive injuries, their consequences, and ser- vices to assist recovery. Arlington (VA): RAND Corporation; 2008.

26. Lippa SM, Fonda JR, Fortier CB, Amick MA, Kenna A, Milberg WP, McGlinchey RE. Deployment-related psychiatric and behavioral conditions and their association with functional disability in OEF/OIF/OND veterans. J Trauma Stress. 2015;28(1):25-33. [PMID:25703936] http://dx.doi.org/10.1002/jts.21979

27. Ross D, Sharp C, Vuchinich RE, Spurrett D. Midbrain mutiny: The picoeconomics and neuroeconomics of disordered gambling. Cambridge (MA): The MIT Press; 2008.

28. McGlinchey RE, Fortier CB, Capozzi SM, Disterhoft JF. Trace eyeblink conditioning in abstinent alcoholic individuals: Effects of complex task demands and prior conditioning. Neuropsychology. 2005;19(2):159-70.

[PMID: 15769200]

http://dx.doi.org/10.1037/0894-4105.19.2.159

Submitted for publication August 18, 2014. Accepted in revised form May 29, 2015.

This article and any supplementary material should be cited as follows:

Fonda JR, Hunt PR, McGlinchey RE, Rudolph JL, Milberg WP, Reynolds MW, Yonan C. Identification of pseudobulbar affect symptoms in Veterans with possible traumatic brain injury. J Rehabil Res Dev. 2015;52(7): 839-50.

http://dx.doi.org/10.1682/JRRD.2014.08.0191

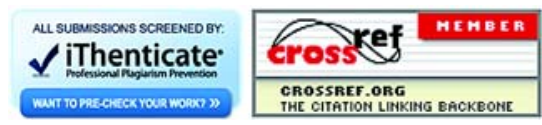


